\title{
ERK signaling mediates long-term low concentration 3,3'-diindolylmethane inhibited nasopharyngeal carcinoma growth and metastasis: An in vitro and in vivo study
}

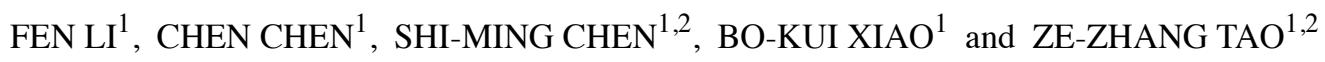 \\ ${ }^{1}$ Otolaryngology-Head and Neck Surgery Research Institute and ${ }^{2}$ Department of Otolaryngology-Head and Neck Surgery II, \\ Renmin Hospital of Wuhan University, Wuhan, Hubei 430060, P.R. China
}

Received September 2, 2015; Accepted October 13, 2015

DOI: $10.3892 /$ or.2015.4428

\begin{abstract}
It is well known that crucifers have antitumor effects and 3,3'-diindolylmethane (DIM) is one of the major bioactive components, and the associated molecular mechanisms in a short-term high-dose manner are widely discussed. However, the antitumor effects of DIM in a long-term low-dose manner in nasopharyngeal carcinoma (NPC) has not been reported yet, as to the potential mechanisms in the human body. In the present study, NPC cells were induced by $20 \mu \mathrm{mol} / 1 \mathrm{DIM}$ for over a month, and the proliferation, apoptosis, migration and in vivo metastasis were investigated. The results showed that DIM significantly reduced the proliferation and migration; however, changes in apoptosis were not observed. In vivo study showed the metastasis was significantly reduced. Compared to the short-term high-dose manner, incomplete similar qualities were observed; next we explored the possible signal pathway revolved, the ERK signaling showed similar changes, while the PI3K/Akt, NF- $\mathrm{BB}, \mathrm{P} 38, \mathrm{JNK}$ pathways were significantly altered in the short-term high-dose manner (our previous study) showed no obvious change, indicating the ERK signaling may be the main effector of DIM.
\end{abstract}

\section{Introduction}

Epigenetic studies have demonstrated that long-term intake of cruciferous vegetables, such as broccoli, carrots and cauliflower, effectively reduced the risk of cancer, as the variety

Correspondence to: Professor Ze-Zhang Tao, Department of Otolaryngology-Head and Neck Surgery II, Renmin Hospital of Wuhan University, Jie-Fang Road 238, Wuhan, Hubei 430060, P.R. China

E-mail: taozezhang@hotmail.com

Dr Chen Chen, Otolaryngology-Head and Neck Surgery Research Institute, Jie-Fang Road 238, Wuhan, Hubei 430060, P.R. China E-mail: chenchen_md@whu.edu.cn

Key words: nasopharyngeal carcinoma, tumor prevention, lymph node metastasis, long-term intake, low concentration, 3,3'-diindolylmethane, ERK of natural anticancer compounds contained in the crucifers could be absorbed in human body. 3,3'-Diindolylmethane (DIM) is one of the most important natural compounds with anticancer properties extracted from crucifers (1). Presently, the natural compounds with anticancer effects causing only slight side-effects are of great interests to medical researchers. Several studies have reported a series of natural compounds, which were extracted from natural materials, with the anticancer effects of inhibiting tumor cell growth and division (2-4), or with anticancer effects of inhibiting tumor metastasis in animal experiments $(5,6)$. Besides, several natural compounds were applied in combination with radiotherapy or chemotherapy in tumor treatment, and enhanced effects of treatments were observed (5,7-9).

DIM was reported as an effective cell proliferation inhibitor and apoptosis inducer in a variety of tumors, such as breast (3), prostate (10,11), pancreatic (8), ovarian (12) and thyroid cancers (13). However, the majority of the studies focused on the short-term killing effects of DIM on tumor cells. Similarly to most of the researches, in our previous study, short-term high-dose manner was designed, and we found that DIM effectively induced cell death in nasopharyngeal carcinoma (NPC) cells both in vitro and in vivo, and no obviously toxic effects were observed on the normal tissues and organs (14). While, whether this type of model could induce similar response in the human body with long-term dietary intake of DIM was unclear, more likely the different key effectors activated in the long-term low-dose manner, thus, in the present study, a long-term low concentration of DIM was chosen to treat the NPC cells, the selection criteria of the concentration referred to several published results $(15,16)$, the detectable concentration of DIM in serum or tissue of human or animal was reported to be $20 \mu \mathrm{mol} / \mathrm{l}$, whereas, no significant inhibitory effects on NPC cell proliferation were observed with $20 \mu \mathrm{mol} / 1 \mathrm{DIM}$ in the short-term response in our previous study (14).

We further explored the antitumor effects of DIM in longterm low-dose manner on NPC cells, as well as the potential targets or effectors playing important roles in the long-term low-dose manner of DIM, which would provide reliable technical indications for clinical use of DIM in long-term low-dose manner. 


\section{Materials and methods}

Reagents. DIM was purchased from Sigma-Aldrich, dissolved in dimethylsulfoxide (both from Sigma-Aldrich St. Louis, MO, USA) and was diluted to $20 \mu \mathrm{M}$ in complete medium (HyClone Corp., Logan, UT, USA). Cell Counting Kit-8 (CCK-8; Dojindo Laboratories, Tokyo, Japan), the antibodies used in the present study were purchased from Cell Signaling Technology Inc. (Danvers, MA, USA).

Cell culture. Human NPC cell lines CNE-2, and 5-8F were maintained in our laboratory, and were stored in liquid nitrogen. The cells were cultured in RPMI-1640 (HyClone Corp.), supplemented with $10 \%$ fetal bovine serum (FBS; Gibco Life Technologies, Carlsbad, CA, USA) and $20 \mu \mathrm{g} / \mathrm{ml}$ antibiotics (ampicilin and kanamycin; Genome Biotechnology, Hangzhou, China), at $37^{\circ} \mathrm{C}, 5 \% \mathrm{CO}_{2}$. Cells in logarithmic growth phase were used in the experiment. The NPC cells CNE-2, and 5-8F were treated with $20 \mu \mathrm{M}$ DIM for over a month then renamed as $\mathrm{CNE}-2 / \mathrm{DIM}^{\mathrm{LL}}$ and 5-8F/DIM ${ }^{\mathrm{LL}}$.

Cell proliferation assay. Cells were seeded into 96-well plates with $1 \times 10^{3}$ cells/well for normal culture. Six double wells were set. After 24, 48, 72 and $96 \mathrm{~h}$ culture, $10 \mu \mathrm{l}$ of CCK- 8 was added in each well, and were incubated at $37^{\circ} \mathrm{C}$ for $1 \mathrm{~h}$, and absorbance value was measured at $450 \mathrm{~nm}$.

Transwell assay. Transwell assay was performed using polycarbonate membrane Transwell (Corning Inc., Corning, NY, USA). The bottom chamber included medium $(0.5 \mathrm{ml})$ containing 5\% FBS. The cell density seeded was $3.0 \times 10^{5} \%$ $\mathrm{ml}$ in the upper chamber, incubated $24 \mathrm{~h}$ at $37^{\circ} \mathrm{C}, 5 \% \mathrm{CO}_{2}$. Membranes were then washed, fixed and stained by Methyl violet (Guge Biotechnology, Wuhan, China). The invasion ability of the cells was determined by counting the cells that had pass through to the lower side of the filter with a microscope.

Flow cytometry. Annexin V/PI apoptosis kit (cat. no. LK-AP101-100; Lianke Biotech Co.,Ltd., Hangzhou,China) was used for the detection. Cells were harvested and washed twice with cold phosphate-buffered saline (Genom Biotechnology). The cells were resuspended in Annexin V binding buffer, and were then stained with $5 \mu \mathrm{l}$ of Annexin V-FITC solution and $10 \mu \mathrm{l}$ of propidium iodide (PI) solution for $15 \mathrm{~min}$ in the dark. Fluorescence was analyzed on a FACSCanto ${ }^{\mathrm{TM}}$ II spectrometer (BD Biosciences, San Jose, CA, USA).

Animal feeding and grouping. The animal experiment was approved by the Ethics Committee of Renmin Hospital of Wuhan University. Forty-eight female BALB/c nude mice (4-6 weeks old) were purchased from Beijing HuaFukang Biological Technology Co. Ltd. (HFK Bioscience, Beijing, China), and underwent adaptive feeding one week before the experiment. The NPC cells at logarithmic phase were collected, resuspended in serum-free medium, then the cell concentration was adjusted to $1 \times 10^{7} / \mathrm{ml}$. Syringe $(1 \mathrm{ml})$ was used for injection of $200 \mu \mathrm{l}$ cell suspension for each animal (14). After the inoculation, the animals were raised for 8 weeks. The short and long diameter of transplanted tumors at 1-8 weeks after the inoculation were measured, 8 weeks after inoculation, animals were sacrificed.

Hematoxylin and eosin $(H \& E)$ staining and immunohistochemistry (IHC). Specimens of transplanted tumors were fixed, embedded and sliced. The H\&E staining was performed following the protocol of the H\&E staining kit (Guge Biotechnology). Then neutral balsam was used for mounting and the section was observed and photographed under a microscope. The IHC was performed with the method of SABC, following the protocol of the IHC kit (Guge Biotechnology).

Western blotting. Cells were harvested and lysed in buffer containing $1 \%$ Nonidet-P40 supplemented with complete protease inhibitor 'cocktail' (Roche) and $2 \mathrm{mM}$ dithiothreitol. Lysates were resolved by $10 \%$ SDS-PAGE, transferred to polyvinylidene defluoride (PVDF) (Immobilon-FL; Millipore, Billerica, MA, USA) membranes and immuno-blotted with primary antibodies. After immunoblotting with the secondary antibody, donkey anti-rabbit immunoglobulin $\mathrm{G}$ (cat. no. 926-3221), the membranes were scanned with Odyssey CLx Infrared Imaging System (both from LI-COR, Lincoln, NE, USA).

Statistical analysis. The values are expressed as the mean \pm SD. Statistical analyses were carried out by one-way ANOVA performed using the SPSS statistical software (SPSS, Inc., Chicago, IL, USA). Probability values of (P-value) $<0.05$ were considered as statistically significant.

\section{Results}

DIM in the long-term low-dose manner significantly reduces the proliferation and migration without influence of apoptosis. To evaluate the effects of long-term low-dose DIM on the proliferation of NPC cells, the proliferation ability was detected with CCK-8 assay. As shown in Fig. 1B, compared to $5-8 \mathrm{~F}$ group, the proliferation rate of $5-8 \mathrm{~F}$ treated with longterm low concentration DIM $\left(5-8 \mathrm{~F} / \mathrm{DIM}^{\mathrm{LL}}\right)$ group decreased by $35 \%(\mathrm{P}<0.05)$, while the proliferation rate of CNE- 2 treated with long-term low concentration DIM (CNE-2/DIM ${ }^{\mathrm{LL}}$ ) group had no significant difference from that of the CNE-2 cell group $(\mathrm{P}>0.05)$. To further explore the effects of longterm low-dose DIM on apoptosis of NPC cells, the apoptotic rates were detected with flow cytometric assay. The results in Fig. $1 \mathrm{C}$ and $\mathrm{D}$ show that the apoptotic rates of $5-8 \mathrm{~F}, 5-8 \mathrm{~F} / \mathrm{DIM}^{\mathrm{LL}}$, CNE-2 and CNE-2/DIM ${ }^{\mathrm{LL}}$ were $(2.7 \pm 0.5 \%),(2.9 \pm 0.4 \%)$, $(1.0 \pm 0.4 \%)$ and $(1.0 \pm 0.6 \%)$, respectively. There was no statistically significant difference between the treatments $(P>0.05)$. In addition, the migration ability was explored to evaluate the effects of long-term low-dose DIM on NPC cells. Transwell assay for migration was performed, as shown in Fig. 1E and F, after $36 \mathrm{~h}$ incubation in the Transwell chamber, the numbers of the membrane-penetrating cells in the four cell groups were $67.3 \pm 8.9,52.4 \pm 10.2,24.8 \pm 6.3$ and $21.2 \pm 7.1$, respectively. Apparently, the number of the membrane-penetrating cells was significantly reduced in the CNE-2/DIM ${ }^{\mathrm{LL}}$ and $5-8 \mathrm{~F} / \mathrm{DIM} \mathrm{ML}^{\mathrm{LL}}$ cell groups as compared to those of the CNE-2 and 5-8F cell groups $(\mathrm{P}<0.01)$. 
A
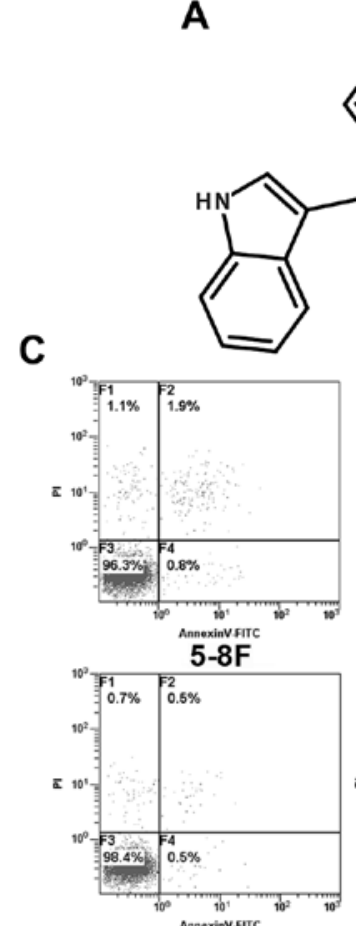

E

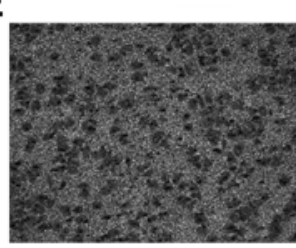

$5-8 \mathrm{~F}$

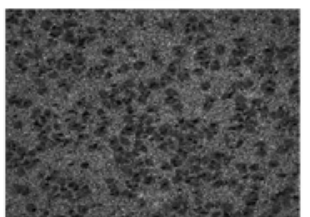

CNE-2

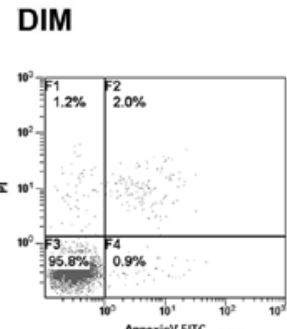

5-8F/DIM ${ }^{\text {neastinf }}$

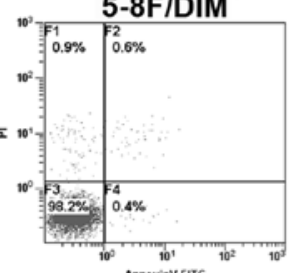

CNE-2/DIM ${ }^{\mathrm{LL}}$

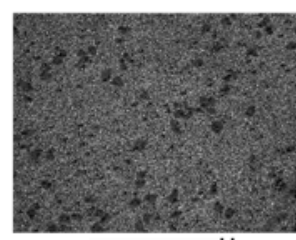

$5-8$ F/DIM ${ }^{\mathrm{LL}}$

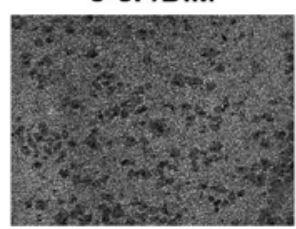

CNE-2/DIM ${ }^{\mathrm{LL}}$
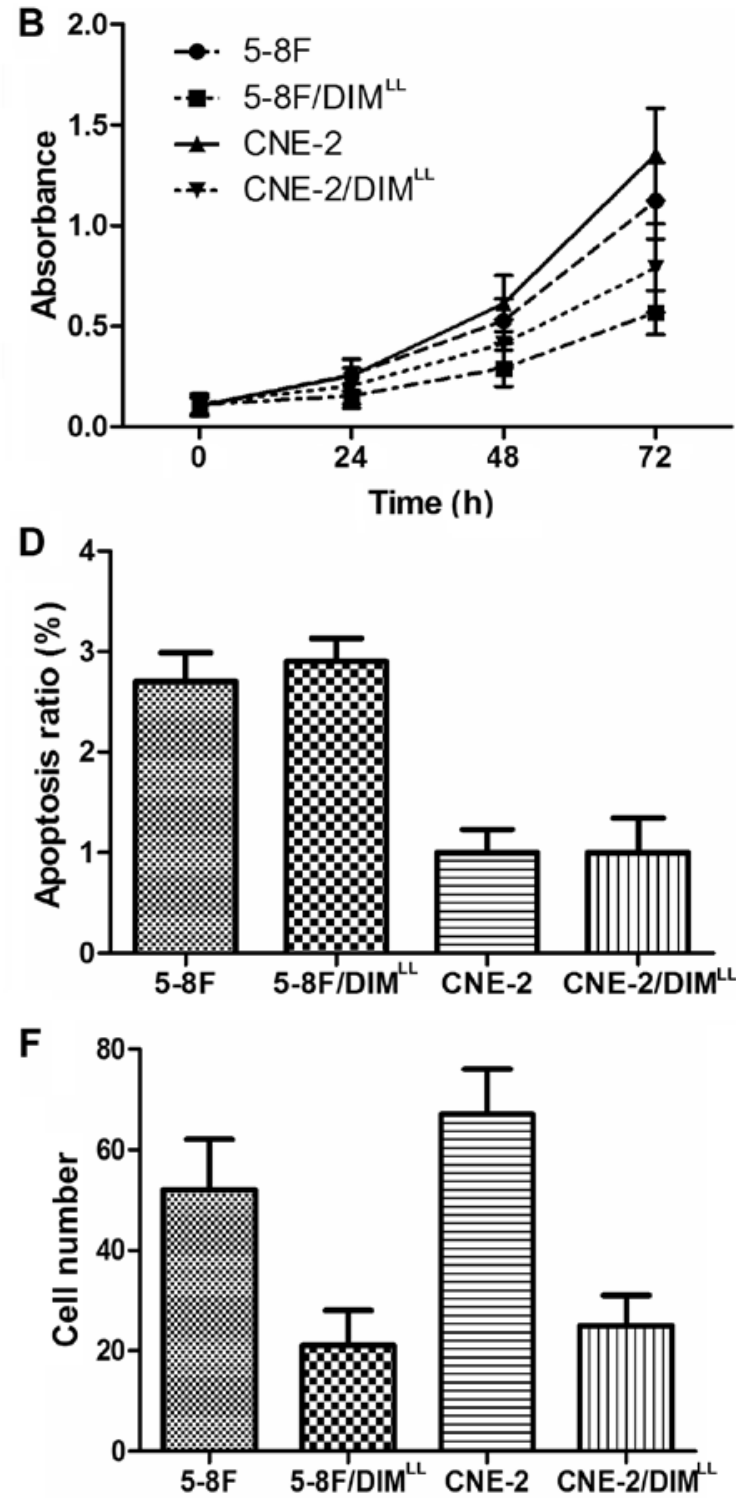

Figure 1. Long-term low-dose of DIM results in the change of proliferative, apoptosis and migration in NPC cells. (A) Molecular formula of DIM; the proliferative capacity was detected by CCK-8 assay (B), compared to 5-8F, 5-8F/DIM ${ }^{\mathrm{LL}}$ group was decreased, while the proliferation rate of CNE-2/DIM ${ }^{\mathrm{LL}}$ group had no apparently difference when compared to CNE-2. The apoptosis rates of $5-8 \mathrm{~F}, 5-8 \mathrm{~F} / \mathrm{DIM}^{\mathrm{LL}}$, CNE-2 and CNE-2/DIM ${ }^{\mathrm{LL}}$ (C and $\mathrm{D}$ ) were detected by a flow cytometric assay, and there was no statistically significant difference between $5-8 \mathrm{~F}$ and $5-8 \mathrm{~F} / \mathrm{DIM}^{\mathrm{LL}}$, similar result was observed between group CNE-2 and CNE-2/DIM ${ }^{\mathrm{LL}}$ (C and D). Transwell assay was chosen for the detection of the migration ability, the numbers of the membrane-penetrating cells of 5-8F/DIM ${ }^{\mathrm{LL}}$, 5-8F, CNE-2/DIM ${ }^{\mathrm{LL}}, \mathrm{CNE}-2$ are shown (E). The histogram of the statistically significant difference between the four groups is shown (F).

DIM in the long-term low-dose manner significantly reduces metastasis in vivo. The in vitro experiments demonstrated that the treatment with long-term low concentration DIM significantly decreased the proliferation and migration in NPC cells. Therefore, an animal experiment was conducted by establishing a subcutaneous xenograft tumor model in nude mice with $5-8 \mathrm{~F}, \mathrm{CNE}-2,5-8 \mathrm{~F} / \mathrm{DIM}^{\mathrm{LL}}$ and CNE-2/ DIM $^{\text {LL }}$ cells. Eight weeks after the NPC cell inoculations, the average volume of the xenograft tumor in the $5-8 \mathrm{~F} /$ $\mathrm{DIM}^{\mathrm{LL}}$ and CNE-2/DIM ${ }^{\mathrm{LL}}$ animal groups were $857.8 \pm 126.1$ and $1034.4 \pm 147.1 \mathrm{~mm}^{3}$, as shown in Fig. 2, respectively. In comparison, the average volume of the xenograft tumor in the $5-8 \mathrm{~F}$ and CNE-2 animal groups were significantly larger, $2032.1 \pm 223.3$ and $2769.1 \pm 241.3 \mathrm{~mm}^{3}$, respectively $(\mathrm{P}<0.05)$. Eight weeks after the NPC cells inoculation, the tumor formation rates of the xenografts in the $5-8 \mathrm{~F}$ and $\mathrm{CNE}-2$

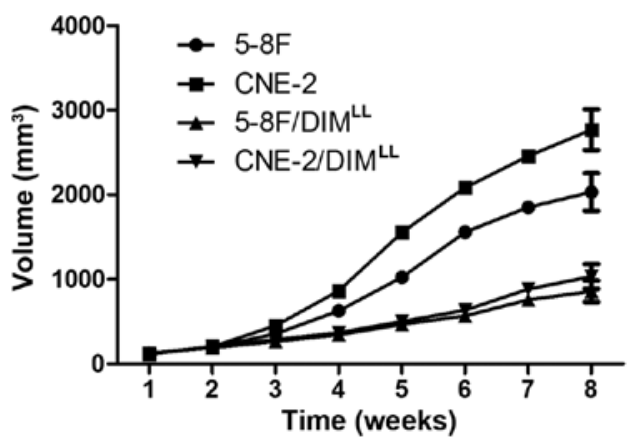

Figure 2. Long-term low-dose of DIM results in the change of proliferative and metastasis in vivo. The average volume of the xenograft tumor from the first week to the 8 week after the NPC cell inoculation of the four groups, the average volume of the xenograft tumor in the 5-8F and CNE-2 animal groups were significantly larger than the $5-8 \mathrm{~F} / \mathrm{DIM} \mathrm{ML}^{\mathrm{LL}}$ and $\mathrm{CNE}-2 / \mathrm{DIM}^{\mathrm{LL}}$ animal groups. 


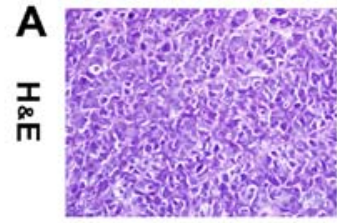

$5-8 \mathrm{~F}$
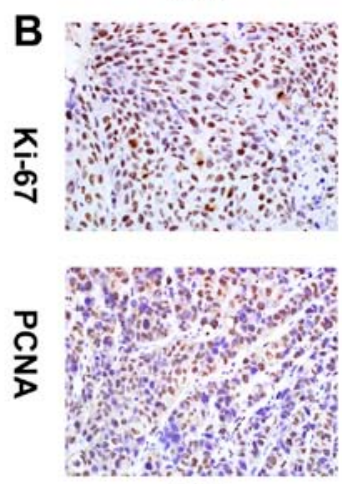

$5-8 \mathrm{~F}$
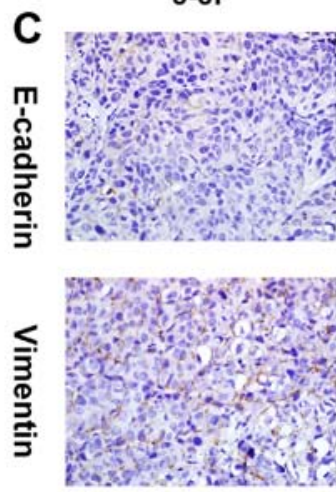

$5-8 \mathrm{~F}$

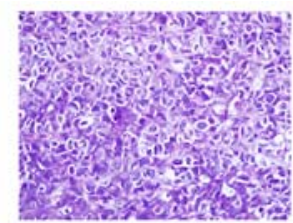

$5-8$ F/DIM ${ }^{\text {LL }}$
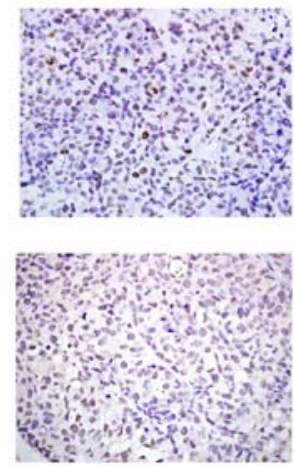

$5-8$ F/DIM ${ }^{L L}$
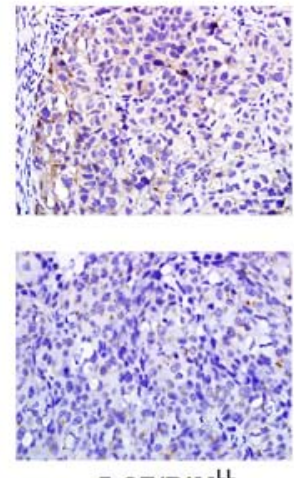

$5-8 \mathrm{~F} / \mathrm{DIM}{ }^{\mathrm{LL}}$

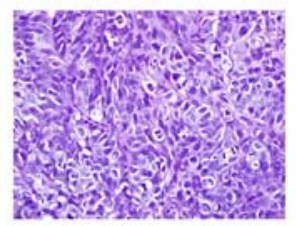

CNE-2
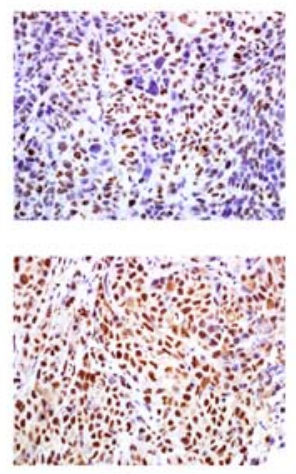

CNE-2
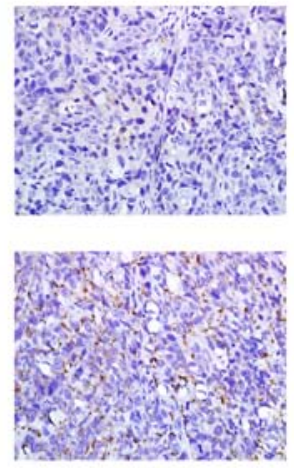

CNE-2

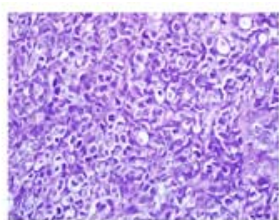

CNE-2/DIM ${ }^{\mathrm{LL}}$
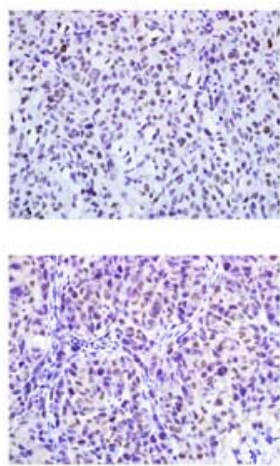

CNE-2/DIM ${ }^{\mathrm{LL}}$
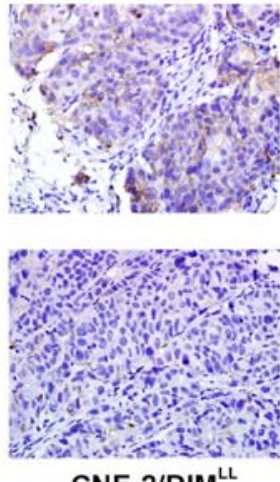

CNE-2/DIM ${ }^{\mathrm{LL}}$

Figure 3. Long-term low-dose of DIM results in the change of proliferation and metastasis-related protein expression in vivo. The xenograft tumors of the four groups were made into tissue paraffin blocks, H\&E staining of tumor tissues are shown (A), IHC assay was chosen for the detection of the expression of proliferation-related molecules Ki-67 and PCNA (B), and the metastasis-related molecules E-cadherin and vimentin (C), the relative expression intensities of Ki-67, PCNA and vimentin were significantly lower $(\mathrm{P}<0.01)$, while the E-cadherin was significantly higher $(\mathrm{P}<0.01)$ in the $5-8 \mathrm{~F} / \mathrm{DIM}{ }^{\mathrm{LL}}$ and $\mathrm{CNE}-2 / \mathrm{DIM}{ }^{\mathrm{LL}}$ groups than the 5-8F and CNE-2 groups.

Table I. Long-term low-dose of DIM resulted in the change of proliferative and metastasis in vivo.

\begin{tabular}{lcrr}
\hline Group & $\begin{array}{c}\text { No. of } \\
\text { mice }\end{array}$ & \multicolumn{1}{c}{$\begin{array}{c}\text { Xenograft } \\
\text { tumor }\end{array}$} & \multicolumn{1}{c}{$\begin{array}{c}\text { Lymph node } \\
\text { metastasis }\end{array}$} \\
\hline $5-8 \mathrm{~F}$ & 12 & $12 / 12(100 \%)$ & $11 / 12(91.7 \%)$ \\
CNE-2 & 12 & $12 / 12(100 \%)$ & $10 / 12(83.3 \%)$ \\
$5-8$ F/DIM & 12 & $9 / 12(75 \%)$ & $2 / 9(22.2 \%)$ \\
CNE-2/DIM & 12 & $10 / 12(83.3 \%)$ & $3 / 10(30 \%)$ \\
\hline
\end{tabular}

DIM, 3,3'-diindolylmethane.

animal groups were $12 / 12$ and $12 / 12$, respectively. The lymph node metastasis rates were $10 / 12$ and $11 / 12$, respectively. In comparison, the $5-8 \mathrm{~F} / \mathrm{DIM}^{\mathrm{LL}}$ and $\mathrm{CNE}-2 / \mathrm{DIM}^{\mathrm{LL}}$ animal groups had a lower tumor formation rates of the xenografts (9/12 and 10/12), and a significantly decreased lymph node metastatic rates $(2 / 9$ and $3 / 10)$, respectively $(\mathrm{P}<0.01)$, and the results are shown in Table I.
Proliferation and metastasis-related protein are altered in the long-term low-dose DIM manner. In the subcutaneous xenograft tumor model of the nude mice, the decrease of proliferation and metastasis of the $5-8 \mathrm{~F}$ and $\mathrm{CNE}-2$ treated with long-term low-dose DIM were observed, then, to further identify the related effectors involved in the effects of antiproliferation and antimetastasis of long-term low-dose DIM, we detected the expression of proliferation related PCNA and $\mathrm{Ki}-67$ as well as metastasis related E-cadherin and vimentin in the xenograft of the nude mice through immunohistochemical assay. As shown in Fig. 3B and C, the relative expression intensities of PCNA, Ki-67 and vimentin were significantly lower $(\mathrm{P}<0.01)$, while the $\mathrm{E}$-cadherin was significantly higher $(\mathrm{P}<0.01)$ in the $5-8 \mathrm{~F} / \mathrm{DIM}^{\mathrm{LL}}$ and $\mathrm{CNE}-2 / \mathrm{DIM}^{\mathrm{LL}}$ groups than the $5-8 \mathrm{~F}$ and CNE-2 groups.

ERK signal pathway is significantly decreased in both the short-term high-dose manner and the long-term low-dose manner of DIM. As indicated above, the NPC cell lines $5-8 \mathrm{~F}$ and CNE-2 underwent significant changes in proliferation, migration, as well as metastasis in the long-term low-dose 


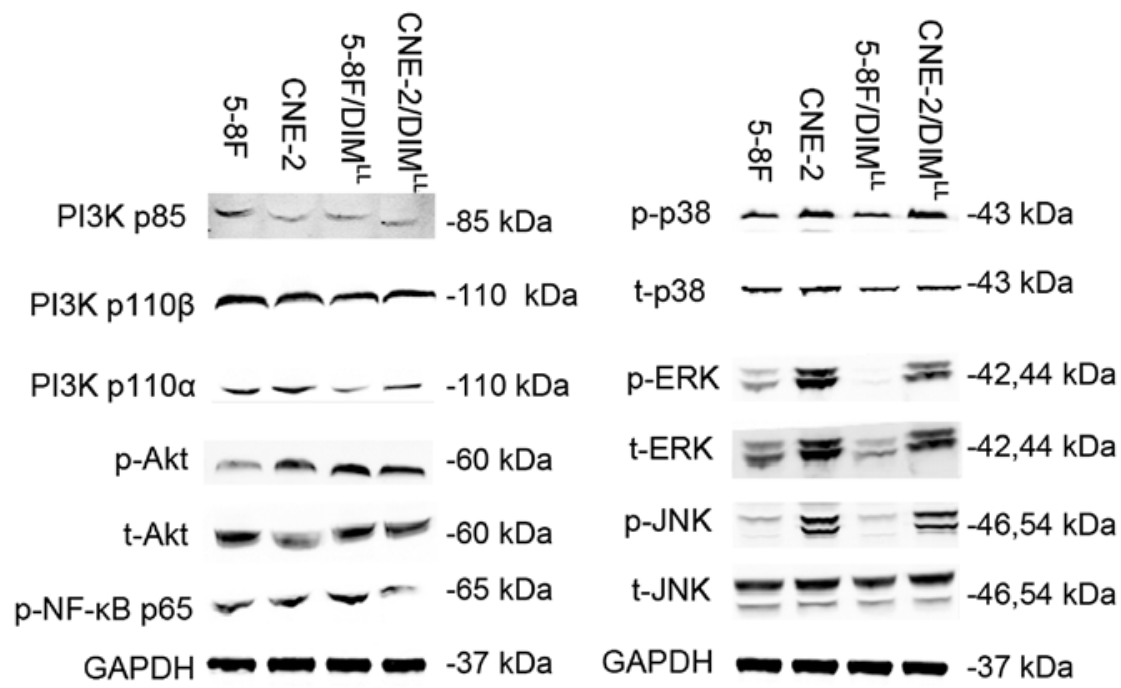

Figure 4. Long-term low-dose of DIM results in the change of proliferation and metastasis-related protein expression in NPC cells. Western blotting was chosen for the detection. The expression of proteins related to the PI3K, NF- $\mathrm{KB}$, MAPK signal pathway were detected, and there were no significant changes between the 5-8F/DIM ${ }^{\mathrm{LL}}, \mathrm{CNE}-2 / \mathrm{DIM}^{\mathrm{LL}}$ and 5-8F, CNE-2 groups in the PI3K, NF- $\mathrm{kB}, \mathrm{P} 38$, JNK pathway, however, the ERK pathway showed a significant decreasing trend in the $5-8 \mathrm{~F} / \mathrm{DIM}^{\mathrm{LL}}, \mathrm{CNE}-2 / \mathrm{DIM}^{\mathrm{LL}}$ groups compared to the $5-8 \mathrm{~F}$ and $\mathrm{CNE}-2$ group.

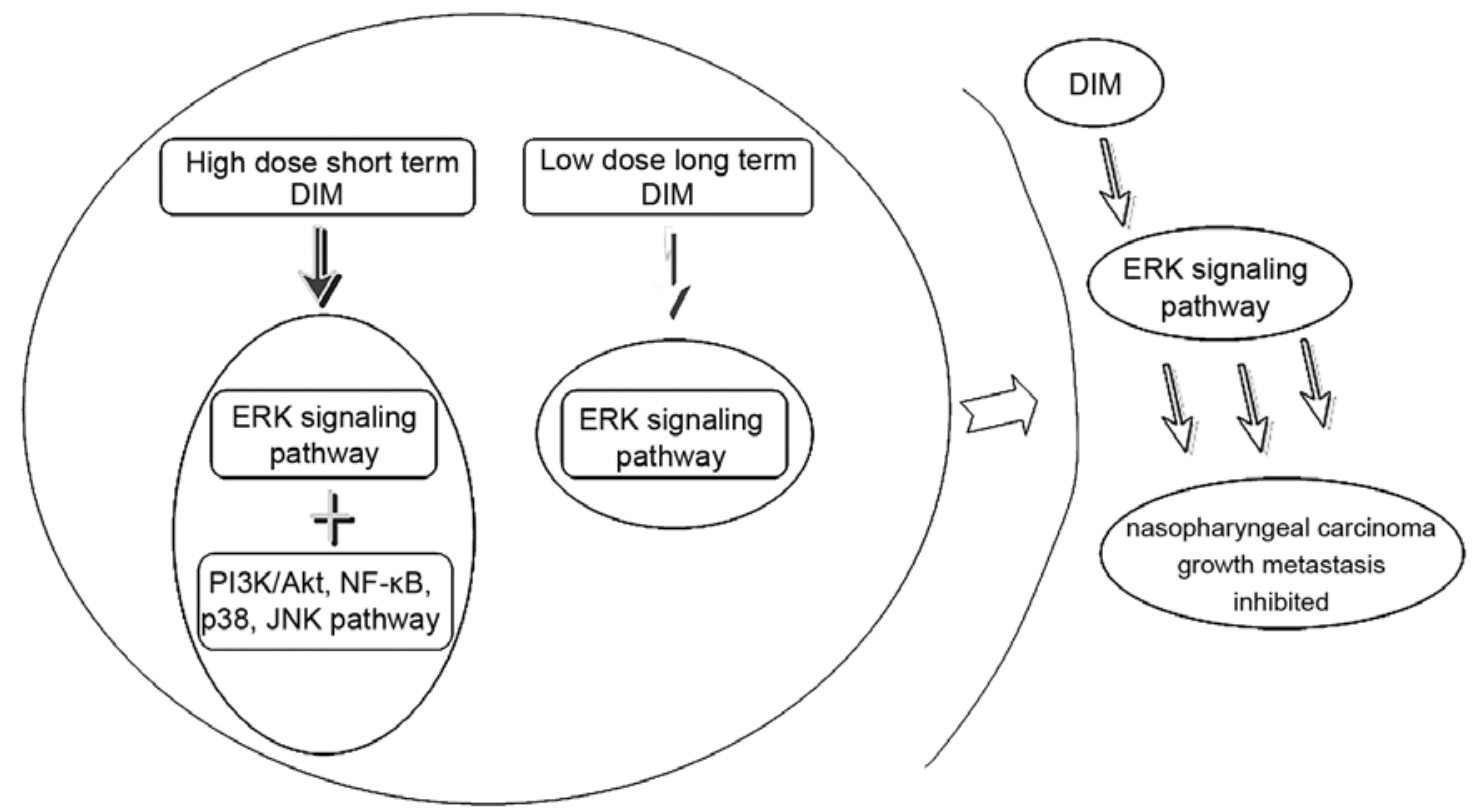

Figure 5. ERK is the major target of DIM in NPC. The ERK signaling pathway was decreased obviously during the long-term low-dose manner of DIM, compared to the short-term high-dose manner of DIM, in which manner that the biological behaviors of NPC cells (CNE-2 and 5-8F) under the treatment of DIM were generally consistent, while the PI3K/Akt, NF-KB, P38 pathway which were significantly reduced, and JNK was significantly increased in the short-term high-dose manner showing no obvious change, thus indicating that the ERK signaling may be the main effector involved in the long-term low-dose manner of DIM treatment.

manner of DIM, therefore, we investigated the key signaling pathways involved in the occurrence and development of NPC, the protein expression in relation to cell proliferation, migration and metastasis was detected by western blotting. Since the short-term high-dose manner of DIM was reported in our previous study (14), the key signaling pathways were detected and compared with the previous results, and the results are shown in Fig. 4, the ERK signaling showed similar changes, while in the short-term high-dose manner (14), the PI3K/Akt, $\mathrm{NF}-\kappa \mathrm{B}, \mathrm{JNK}$ pathways which were significantly reduced, and the P38 pathway, which was significantly increased, showed no obvious change, indicating the ERK signaling may be the main effector involved in the long-term low-dose manner of DIM.

\section{Discussion}

DIM is a natural compound extracted from cruciferous vegetables, and widely recognized for its antitumor effects. Several in vitro studies had reported the pro-apoptosis effects in tumor 
cells in short-term high-dose manner of DIM (2,5,8-10). In our previous study, short-term high concentration of DIM effectively inhibited the proliferation and apoptosis of NPC cells in a dosedependent manner. Moreover, the results of animal experiments showed that DIM had certain effects on NPC prevention and inhibition, including slowing down the tumor progression and reducing the incidence of tumor metastasis (14). In the present study, we raised the suggestion that, treatment with high-dose of DIM would induce an acute or transient response, as a result, the experiments based on the high-dose DIM may only show an acute or transient response in the body, whereas the continued dietary intake of DIM resulted in a relatively steady-state, the response in vivo would be different, therefore the long-term low-dose was designed for the study.

Long-term intake of edible crucifers effectively prevents the occurrence of specific tumors. The possible mechanism is thought to be that, a certain dose of DIM was persistently identified in the blood in the condition of sustaining intake of crucifers, the early mutations which resulted in the proliferation induction or the apoptosis inhibition of the tumor cells were thereby effectively inhibited. Studies have shown that colon cancer spontaneously occurred in heterozygous TRAMP mice, while oral administration of DIM significantly decreased its incidence and alleviated the severity of colon lesions in the transgenic mice. These findings indicated that DIM effectively inhibited the occurrence of colon cancer and prevented its progression (17). In another experiment, Howells et al found that DIM was detectable in the blood samples with a concentration up to $20 \mu \mathrm{mol} / 1(16,18)$. Similarly, Moiseeva et al (19) found that a continued treatment with low concentration of DIM significantly changed the specific gene expression of the breast cancer MD-MBA-231 cells and affected their biological behavior, such as the doubling time, motility and ability to repair DNA damage. Therefore, in the present study, we conducted in vivo and in vitro experiments in order to examine the effect of long-term low-dose DIM treatment on the NPC cells; moreover, the related underlying molecular mechanism was further investigated.

Our previous results showed that after 48-72 h of treatment with $20 \mu \mathrm{mol} / 1$ of DIM, no obvious changes were observed in the NPC cells, neither proliferation inhibition nor apoptosis induction (14), however, the continued treatments with $20 \mu \mathrm{mol} / 1 \mathrm{DIM}$ for over a month resulted in significant changes in proliferation, migration and metastasis. While, there was a slight difference between the two NPC cell lines. For example, after being treated with $20 \mu \mathrm{mol} / 1$ of DIM for over 30 days, the $5-8 \mathrm{~F}$ cell line had a significant reduction in proliferation ability, migration and metastasis, however, the CNE-2 cell line had no evident decrease in the proliferation ability, but showed a decreased ability for migration and metastasis. Such variations could be related to the differences of gene expression profiles or epigenetic properties between various cancer cell lines.

The signaling pathways PI3K/Akt, MAPK and NF- $\mathrm{kB}$, which played an important role in the occurrence, pathogenesis and metastasis processes of NPC (20-24), were reported in regulation of cell proliferation, migration and metastasis. In addition, they were detected by western blot assay in the study, aimed to explore the possible targets of DIM $(20 \mu \mathrm{mol} / 1$, over a month) in NPC. It turned out that the ERK pathway was significantly decreased during the treatment, while the other primary signal pathways were not obviously changed. Compared to the short-term high-dose manner in our previous study, the expression changes of relevant signaling pathway proteins differed.

ERK signaling pathway was vital in vivo, a number of important biological process were under the regulation of ERK signaling pathway, such as proliferation, differentiation, apoptosis, cancerization and other biological reaction. The ERK1/2 was located in the cytosol in an inactivated state, when phosphorylated to activate, translocated from the cytosol to the nucleus, and affected the multiple biological processes through the regulation of the activity of transcription factors. ERK was reported activated in a majority of NPC (25-27). Since we observed that the ERK signaling showed similar changes, while the PI3K/Akt, NF- $\mathrm{BB}, \mathrm{P} 38$ and JNK pathways which were significantly changed in the short-term high-dose manner (14) showed no obvious change, indicated the ERK signaling may be the main effector involved in the long-term low-dose manner of DIM treatment. Based on the differences of the activated signals (Fig. 5), we speculated that the PI3K/ Akt, NF- $\mathrm{kB}, \mathrm{P} 38$, JNK pathways which were activated/inactivated during the short-term high-dose manner of DIM were only thought to be the drug toxic reaction of the high-dose DIM, and also considered to be the reason that apoptosis increased in the high-dose of DIM, by contrast, apoptosis was not changed in the low-dose of DIM, these results confirmed that ERK may be the real target of DIM in the long-term lowdose treatment of DIM in NPC.

In conclusion, a long-term low-dose DIM treatment (20 $\mu \mathrm{mol} / \mathrm{l})$ inhibited the proliferation, migration, as well as the in vivo metastasis in NPC cells, further, the ERK signaling pathway may be the main effector in the long-term low-dose DIM. Our results provide evidence that the use of long-term low-dose DIM suppresses the activation of the ERK pathway, which would add support for the use of DIM in preclinical and clinical settings in the management of NPC patients.

\section{Acknowledgements}

The present study was supported by the grants from the National Natural Science Foundation of China (no. 81372880), the Independent Research Project of Wuhan University (nos. 2042014kf0184 and 2042014kf0119), the Doctoral Program of Higher Education Research Fund (nos. 20130141120093 and 20110141110062), and the Natural Science Foundation of Hubei Province (no. 2012FFA045).

\section{References}

1. Safe S, Papineni S and Chintharlapalli S: Cancer chemotherapy with indole-3-carbinol, bis(3'-indolyl)methane and synthetic analogs. Cancer Lett 269: 326-338, 2008.

2. Chinnakannu K, Chen D, Li Y, Wang Z, Dou QP, Reddy GP and Sarkar FH: Cell cycle-dependent effects of 3,3'-diindolylmethane on proliferation and apoptosis of prostate cancer cells. J Cell Physiol 219: 94-99, 2009.

3. Jin Y, Zou X and Feng X: 3,3'-Diindolylmethane negatively regulates $\mathrm{Cdc} 25 \mathrm{~A}$ and induces a $\mathrm{G} 2 / \mathrm{M}$ arrest by modulation of microRNA 21 in human breast cancer cells. Anticancer Drugs 21: 814-822, 2010.

4. Wang YQ, Chen C, Chen Z, Xu Y, Wang Y, Xiao BK, Chen SM and Tao ZZ: Indole-3-carbinol inhibits cell proliferation and induces apoptosis in Hep-2 laryngeal cancer cells. Oncol Rep 30: 227-233, 2013. 
5. Ahmad A, Kong D, Wang Z, Sarkar SH, Banerjee S and Sarkar FH: Down-regulation of uPA and uPAR by 3,3'-diindolylmethane contributes to the inhibition of cell growth and migration of breast cancer cells. J Cell Biochem 108: 916-925, 2009.

6. Kim EJ, Shin M, Park H, Hong JE, Shin HK, Kim J, Kwon DY and Park JH: Oral administration of 3,3'-diindolylmethane inhibits lung metastasis of 4T1 murine mammary carcinoma cells in BALB/c mice. J Nutr 139: 2373-2379, 2009.

7. Ali S, Banerjee S, Ahmad A, El-Rayes BF, Philip PA and Sarkar FH: Apoptosis-inducing effect of erlotinib is potentiated by 3,3'-diindolylmethane in vitro and in vivo using an orthotopic model of pancreatic cancer. Mol Cancer Ther 7: 1708-1719, 2008.

8. Banerjee S, WangZ,Kong DandSarkarFH:3,3'-Diindolylmethane enhances chemosensitivity of multiple chemotherapeutic agents in pancreatic cancer. Cancer Res 69: 5592-5600, 2009.

9. Rahman KMW, Banerjee S, Ali S, Ahmad A, Wang Z, Kong D and Sakr WA: 3,3'-Diindolylmethane enhances taxotere-induced apoptosis in hormone-refractory prostate cancer cells through survivin down-regulation. Cancer Res 69: 4468-4475, 2009.

10. Smith S, Sepkovic D, Bradlow HL and Auborn KJ: 3,3'-Diindolylmethane and genistein decrease the adverse effects of estrogen in LNCaP and PC-3 prostate cancer cells. J Nutr 138: 2379-2385, 2008.

11. Vivar OI, Lin CL, Firestone GL and Bjeldanes LF: 3,3'-Diindolylmethane induces a $\mathrm{G}_{1}$ arrest in human prostate cancer cells irrespective of androgen receptor and p53 status. Biochem Pharmacol 78: 469-476, 2009.

12. Hsu EL, Chen N, Westbrook A, Wang F, Zhang R, Taylor RT and Hankinson O: CXCR4 and CXCL12 down-regulation: A novel mechanism for the chemoprotection of 3,3'-diindolylmethane for breast and ovarian cancers. Cancer Lett 265: 113-123, 2008.

13. Rajoria S, Suriano R, George A, Shanmugam A, Schantz SP, Geliebter J and Tiwari RK: Estrogen induced metastatic modulators MMP-2 and MMP-9 are targets of 3,3'-diindolylmethane in thyroid cancer. PLoS One 6: e15879, 2011.

14. Chen C, Chen SM, Xu B, Chen Z, Wang F, Ren J, Xu Y, Wang Y, $\mathrm{Xiao} \mathrm{BK}$ and Tao ZZ: In vivo and in vitro study on the role of 3,3'-diindolylmethane in treatment and prevention of nasopharyngeal carcinoma. Carcinogenesis 34: 1815-1821, 2013

15. Manach C, Williamson G, Morand C, Scalbert A and Rémésy C: Bioavailability and bioefficacy of polyphenols in humans. I. Review of 97 bioavailability studies. Am J Clin Nutr 81 (Suppl 1): 230S-242S, 2005

16. Howells LM, Moiseeva EP, Neal CP, Foreman BE, Andreadi CK, Sun YY, Hudson EA and Manson MM: Predicting the physiological relevance of in vitro cancer preventive activities of phytochemicals. Acta Pharmacol Sin 28: 1274-1304, 2007.

17. Cho HJ, Park SY, Kim EJ, Kim JK and Park JH: 3,3'-Diindolylmethane inhibits prostate cancer development in the transgenic adenocarcinoma mouse prostate model. Mol Carcinog 50: $100-112,2011$
18. Heath EI, Heilbrun LK, Li J, Vaishampayan U, Harper F, Pemberton P and Sarkar FH: A phase I dose-escalation study of oral BR-DIM (BioResponse 3,3'- Diindolylmethane) in castrateresistant, non-metastatic prostate cancer. Am J Transl Res 2: 402-411, 2010.

19. Moiseeva EP, Almeida GM, Jones GDD and Manson MM: Extended treatment with physiologic concentrations of dietary phytochemicals results in altered gene expression, reduced growth, and apoptosis of cancer cells. Mol Cancer Ther 6: 3071-3079, 2007.

20. Koon HK, Chan PS, Wong RNS, Wu ZG, Lung ML, Chang CK and Mak NK: Targeted inhibition of the EGFR pathways enhances Zn-BC-AM PDT-induced apoptosis in well-differentiated nasopharyngeal carcinoma cells. J Cell Biochem 108: $1356-1363,2009$

21. Horikawa T, Yoshizaki T, Kondo S, Furukawa M, Kaizaki Y and Pagano JS: Epstein-Barr Virus latent membrane protein 1 induces Snail and epithelial-mesenchymal transition in metastatic nasopharyngeal carcinoma. Br J Cancer 104: 1160-1167, 2011.

22. Qu C, Liang Z, Huang J, Zhao R, Su C, Wang S, Wang X, Zhang R, Lee MH and Yang H: MiR-205 determines the radioresistance of human nasopharyngeal carcinoma by directly targeting PTEN. Cell Cycle 11: 785-796, 2012

23. Wong VC, Chen H, Ko JM, Chan KW, Chan YP, Law S, Chua D, Kwong DL, Lung HL, Srivastava G, et al: Tumor suppressor dualspecificity phosphatase 6 (DUSP6) impairs cell invasion and epithelial-mesenchymal transition (EMT)-associated phenotype. Int J Cancer 130: 83-95, 2012.

24. Yang F, Qian XJ, Qin W, Deng R, Wu XQ, Qin J, Feng GK and Zhu XF: Dual phosphoinositide 3-kinase/mammalian target of rapamycin inhibitor NVP-BEZ235 has a therapeutic potential and sensitizes cisplatin in nasopharyngeal carcinoma. PLoS 8: e59879, 2013

25. Xie YQ, Wu XB and Tang SQ: Curcumin treatment alters ERK-1/2 signaling in vitro and inhibits nasopharyngeal carcinoma proliferation in mouse xenografts. Int J Clin Exp Med 7: 108-114, 2014.

26. Chen LC, Liu HP, Li HP, Hsueh C, Yu JS, Liang CL and Chang YS: Thymidine phosphorylase mRNA stability and protein levels are increased through ERK-mediated cytoplasmic accumulation of hnRNP K in nasopharyngeal carcinoma cells. Oncogene 28: 1904-1915, 2009.

27. Peng C, Liu HY, Zhou M, Zhang LM, Li XL, Shen SR and Li GY: BRD7 suppresses the growth of nasopharyngeal carcinoma cells (HNE1) through negatively regulating beta-catenin and ERK pathways. Mol Cell Biochem 303: 141-149, 2007. 